Article

\title{
Integrated Tomato Picking and Distribution Scheduling Based on Maturity
}

\author{
Anqi Zhu ${ }^{1}$, Bei Bian ${ }^{1}$, Yiping Jiang ${ }^{1, *(1)}$ and Jiaxiang $\mathrm{Hu}^{2,3}$ \\ 1 School of Information Management, Nanjing Agricultural University, Nanjing 210031, China; \\ zhuanqiii@163.com (A.Z.); 2018112037@njau.edu.cn (B.B.) \\ 2 College of Economics and Management, Nanjing Agricultural University, Nanjing 210095, China; \\ hujiaxiang@njau.edu.cn \\ 3 China Center for Food Security Studies, Nanjing Agricultural University, Nanjing 210095, China \\ * Correspondence: ypjiang@njau.edu.cn; Tel.: +86-25-5860-6573
}

Received: 17 August 2020; Accepted: 22 September 2020; Published: 25 September 2020

\begin{abstract}
Agriproducts have the characteristics of short lifespan and quality decay due to the maturity factor. With the development of e-commerce, high timelines and quality have become a new pursuit for agriproduct online retailing. To satisfy the new demands of customers, reducing the time from receiving orders to distribution and improving agriproduct quality are significantly needed advancements. In this study, we focus on the joint optimization of the fulfillment of online tomato orders that integrates picking and distribution simultaneously within the context of the farm-to-door model. A tomato maturity model with a firmness indicator is proposed firstly. Then, we incorporate the tomato maturity model function into the integrated picking and distribution schedule and formulate a multiple-vehicle routing problem with time windows. Next, to solve the model, an improved genetic algorithm (the sweep-adaptive genetic algorithm, S-AGA) is addressed. Finally, we prove the validity of the proposed model and the superiority of S-AGA with different numerical experiments. The results show that significant improvements are obtained in the overall tomato supply chain efficiency and quality. For instance, tomato quality and customer satisfaction increased by $5 \%$ when considering the joint optimization, and the order processing speed increased over $90 \%$ compared with traditional GA. This study could provide scientific tomato picking and distribution scheduling to satisfy the multiple requirements of consumers and improve agricultural and logistics sustainability.
\end{abstract}

Keywords: tomato; maturity; picking; farm-to-door distribution; joint optimization; sweep-adaptive genetic algorithm

\section{Introduction}

With the development of e-commerce platforms, the farm-to-door model has received critical attention in recent years. It can not only avoid the redundant part of distribution effectively but can also reduce the loss in the handling of agriproducts during the process. Merchants gain more profit by providing a value-added service for agriproducts through picking, packing, warehousing, and transportation [1], and consumers get their orders faster in the meanwhile. However, the quality of agriproducts will decline after picking due to the property of maturation. Time will increase quality decay after picking and distribution, affecting consumer satisfaction. Fresh agriproducts, represented by tomatoes, have a short lifespan and quality decay nature. Respiration affects the food flavor, and the agriproducts even lose commercial value during storage and transportation [2], resulting in huge losses [3]. Therefore, scheduling a proper tomato picking and distribution scheme so as to reduce quality decay, improve the sustainability of the tomato supply chain, and satisfy consumers' multiple requirements has been an essential problem for tomato trading in the farm-to-door context. 
Current research on the agriproduct supply chain can be mainly grouped into four functional areas: production, harvesting, storage, and distribution [4]. Omar et al. [5] presented a stochastic tactical planning model for the production and distribution of fresh agricultural products, and it showed significant improvements in the planning recommendations. Amalia and Villalobos [6] focused on agricultural planning (ARP) for farmers' fields and introduced an evolutionary hybrid neighborhood search (EHNS) to solve the problem, reducing an average of $10.68 \%$ in nonworking distance for $56 \%$ of cases. Javier et al. [7] addressed a harvest problem by proposing a mixed-integer linear programming model that includes costs related to the fruit not reaching maturity and the number of harvest days and developed a greedy randomized adaptive search procedure (GRASP) metaheuristic method to solve the problem. Graf [8] proposed a method for harvest planning based on the coupling of crop assignment with vehicle routing and derived integer-programming-based exact algorithms that were unable to tract a large number of fields but maintained large-scale applicability. Ali et al. [9] formulated infield logistics planning for crop-harvesting operations to improve the efficiency of the process. Kamal et al. [10] considered the organizational structure of agricultural systems in the logistics of harvesting agricultural crops and developed a model to plan the movement of the crop from farm to processing plant. Three significant agricultural systems in the United States were also described in this paper to demonstrate the model. Liu et al. [11] addressed decisions for optimal purchase and inventory retrieval quantities for perishable seasonal agricultural products, proposed a single-product finite-period inventory model, and compared the performance of two policies, increasing the expected profit and reducing the expected loss in the meanwhile. Wang et al. [12] proposed a multiobjective vehicle-routing problem with time windows dealing with perishability (MO-VRPTW-P) and applied a two-phase heuristic algorithm based on the Pareto variable neighborhood search genetic algorithm considering temporal-spatial distance (STVNS-GA) to solve the model. Fan [13] focused on the vehicle-routing problem, with simultaneous pickup and delivery based on customer satisfaction (VRPSPDCS) and used customer satisfaction to reflect the service quality, which is inversely proportional to the waiting time for the vehicle. Li et al. [14] perfected the calculation of the cost of cold chain delivery by the energy cost generated due to cooling and the damage cost accumulated due to changes in time and temperature. In this study, there are large quantities of literature considering the production, harvesting, storage, and distribution scheduling of agriproducts separately to reduce the logistics cost and quality decay. Very few studies have formulated the joint optimization of the supply chain functional areas like Omar [5]. However, the integrated optimization of the agriproduct supply chain should pay more attention to reducing quality decay and agrilogistics costs. Picking and distribution are two highly related parts to the scheduling of the tomato supply chain. Therefore, we propose a tomato maturity model and incorporate it into the integrated picking and distribution of tomato retailing within the context of a farm-to-door model, which can improve the overall supply chain efficiency and sustainability.

Logistics enterprises generally use a manual experience to arrange picking scheduling without the necessary theoretical basis support. The integrated tomato picking and distribution schedule provides an effective method to reduce logistics cost and quality decay in the tomato supply chain. This decision-making problem states the tomato picking sequence, the distribution routes according to customer demand, geographical location, and time windows, and the quality deterioration of tomatoes over time. In this paper, we focus on two questions: (1) how to determine tomato maturity, and (2) how to make an integrated tomato picking and distribution schedule with time windows.

Our contributions in this study include three aspects: (1) presenting a quantitative way to model tomato maturity as an exponential function with a firmness indicator, (2) proposing a multiple-vehicle routing problem with time windows to formulate an integrated tomato picking and distribution schedule, and (3) addressing a sweep-adaptive genetic algorithm (S-AGA) to solve the model and carry out small-scale and large-scale numerical experiments to prove the validity of the proposed model.

Our paper is organized as follows. In Section 2, we propose a tomato maturity model with a firmness indicator. In Section 3, we build an integrated tomato picking and distribution model. 
In Section 4, we present a sweep-adaptive genetic algorithm to solve the proposed model. In Section 5, we report the numerical results and the comparison of computer performance between the genetic algorithm (GA) and the sweep-adaptive genetic algorithm (S-AGA). In Section 6, we discuss the conclusions and future works.

\section{Tomato Maturity Model}

Maturity is an important indicator to measure tomato quality [15], so, in this study, we propose a tomato maturity model to be the measurement index of quality. Nowadays, the measurement of tomato maturity and quality mainly relies on artificial sensory assessment [16], physicochemical analysis [17], and nondestructive testing technology [18]. Tomato maturity is a complex physiological and biochemical process, during which a series of changes will take place. The main characteristic is the deterioration of firmness. Therefore, firmness is an important indicator of fruit maturity [19]. According to the study and measurement of Holt [20], Patrick, and Marie [21] and Zsom et al. [22] on the nondestructive testing of tomato firmness, we can take firmness as a critical indicator to measure tomato maturity. Although some studies have added maturity to the research on logistics distribution $[5,23,24]$, most of them only considered the quality change of products in the process of distribution and ignored the change in the process of large-scale orders and long-time picking. Moreover, the rate of ripeness also influences the picking process. The picking process and distribution process influence and restrict each other. Therefore, this study considers integrated tomato picking and distribution scheduling based on maturity.

The tomato maturity can be divided into six stages according to six ripening periods [25], and tomatoes mature naturally after being picked during the six stages. Though the duration of tomato transformation from one stage to the next is different, its surface characteristics and physicochemical properties have a certain trend [15]. Therefore, tomato maturity can be expressed by a certain character of tomatoes.

Firmness is an important indicator to measure tomato maturity, and it shows a very obvious change with time [19]. In this study, partial least-square is used to establish the maturity model. The Dalian Traditional No.1 tomato is selected as the experimental material and measured at different maturity stages. We stored the samples in a constant temperature and humidity box at $25{ }^{\circ} \mathrm{C}$ and employed the professional US FTC TMS-Pro Texture analyzer with a cylindrical probe to get the data of tomato firmness. Six representative data points were selected in each stage. We have chosen the maximum data points (Data 1), the middle data points (Data 2), and the minimum data points (Data 3) of each stage, respectively, to represent the firmness of tomato maturity and the changes of firmness on different days were obtained, as shown with the dotted line in Figure 1. It can be seen that firmness is negatively correlated with time. The SPSS statistics 19.0 package is applied for data analysis.

Shiue [26] pointed out that the deterioration rate of perishable items with a lifespan is usually expressed in exponential form. Therefore, this study uses the exponential form to carry out the maturity model. The tomato maturity model is shown in Equation (1). Here, $t$ denotes the time since picking; $R(t)$ denotes tomato firmness at different maturity stages. Visualize the fitted function, as shown in the solid curve in Figure 1, being close to the variation trend of the data curve. Numerical examination is shown in Table 1. In the model, $t$ is selected to produce an adjusted goodness of fit ( $R$-square), $R^{2}=0.977$. The significance test $(p=0.000)$ and the $F$-test are both passed $(F=171.914<224.583)$. It can be concluded that there is an extremely significant correlation between time and firmness. Each specific range of firmness corresponds to a different tomato maturity stage, guiding the practical tomato picking process. In this study, we use the principle of proximity to classify tomato maturity based on firmness. For example, Maturity 8 corresponds to the tomato firmness interval $(31,35]$.

$$
R(t)=59.726 e^{-0.047 t}
$$




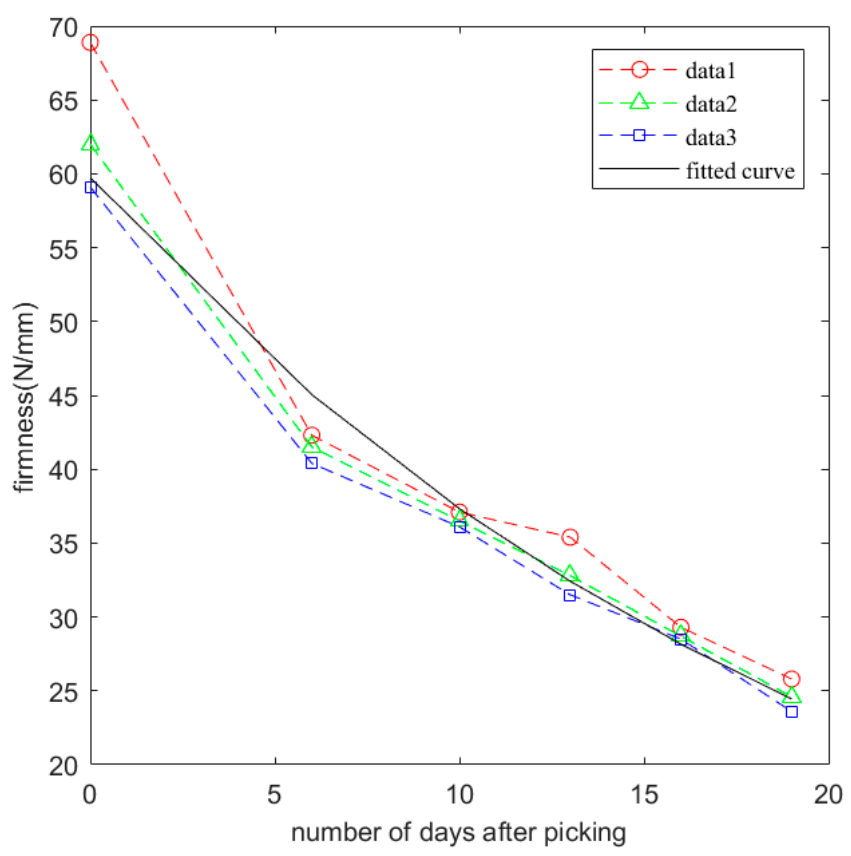

Figure 1. Measurement and fitting curve of tomato firmness with time.

Table 1. Numerical examination.

\begin{tabular}{ccccc}
\hline R-Square & F & DOF 1 & DOF 2 & Significance \\
\hline 0.977 & 171.914 & 1 & 4 & 0.000 \\
\hline
\end{tabular}

\section{Model Formulation}

We considered an integrated tomato picking and distribution schedule based on maturity and modeled this problem as a multiple-vehicle routing problem with time windows (m-VRPTW). The objective is to minimize the total cost, including distribution cost and discipline cost.

\subsection{Problem Description}

Farm-to-door distribution is a superior model to reduce the logistics process and costs because only suppliers and customers participate in the whole agriproduct supply chain. However, in such a context, the supply chain will meet a lot of challenges on account of repaid delivery and high customer requirements. The farm-to-door distribution process of agriproduct online retailing contains order processing, picking, packaging, vehicle assignment, and distribution. In this study, we illustrate the picking, vehicle assignment, and distribution to formulate an integrated tomato picking and distribution schedule based on maturity, as shown in Figure 2. It contains one tomato production base, one packaging area, various consumers located in different places, and multiple types of vehicles. The tomato production base receives numerous orders $O=\left\{O_{1}, O_{2}, \ldots, O_{i}\right\}$, including online and offline orders within the specified scope. After order processing, tomatoes are picked, packed, and delivered directly to customers $N=\{1,2, \ldots, d\}$ with the time required windows $\left(E T_{i}, L T_{i}\right)$, using vehicle $K=\left\{k_{1}, k_{2}, \ldots, k_{|k|}\right\}$ of $M=\left\{m_{1}, m_{2}, \ldots, m_{|m|}\right\}$. Optimal vehicle routings are programmed after considering tomato maturity $R(t)$ and time windows $\left(E T_{i}, L T_{i}\right)$. The tomato maturity function, order details including coordinates, demand, and the type and load of vehicles in the production base are known.

To clarify the scope of the application of this paper, some assumptions are proposed for the model system: (1) the orders are delivered by the same vehicle along one route, and each customer is served only once; (2) we ignore the customer service time and order processing time; (3) the variable shipping cost is proportional to the driving distance; (4) the traffic condition is good, no blocking or situation will 
be met; (5) each vehicle delivers orders from the tomato production base and returns to it immediately after distribution; (6) the rule of tomato picking is that one vehicle picks another vehicle after it has been picked, and each vehicle will be used as transport at once after it has been picked.

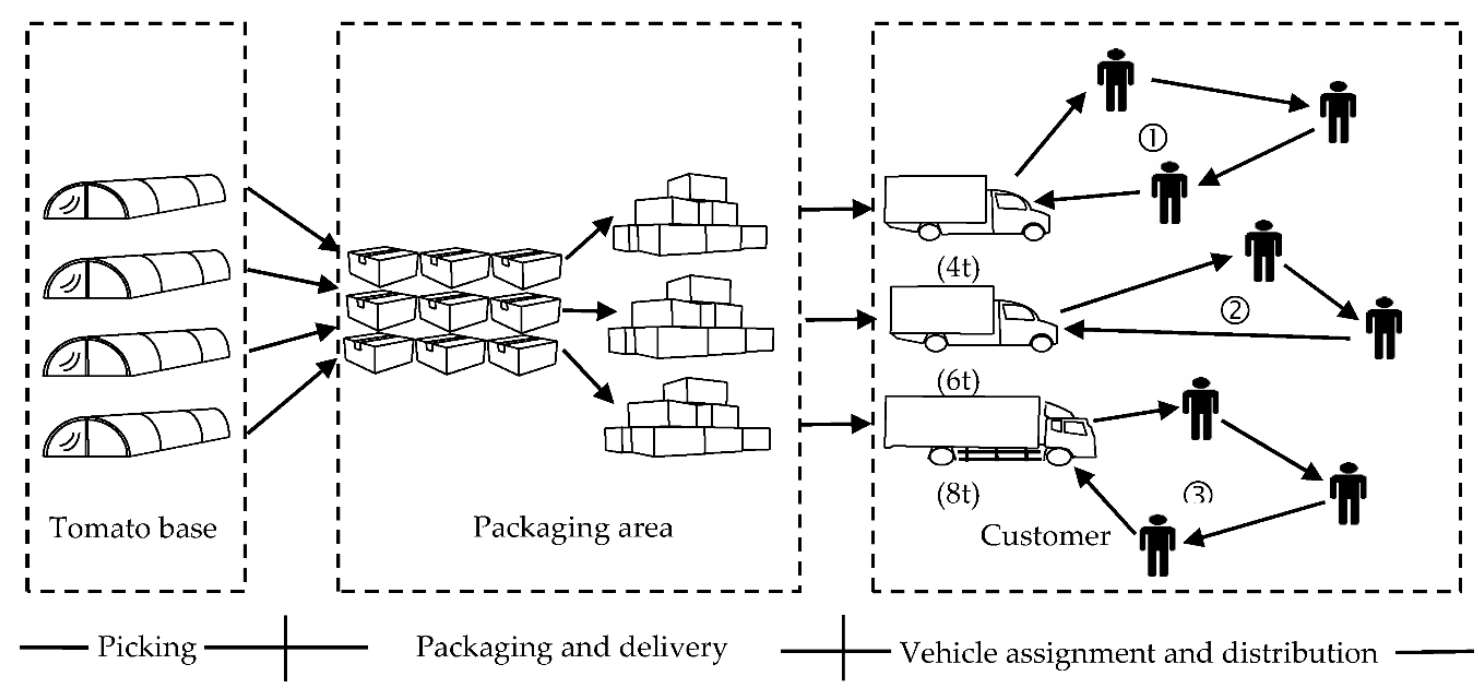

Figure 2. Tomato picking and distribution process.

The main notations are shown in Table 2. The decisions to be taken in this model are as follows: (1) how many of each type of vehicle should be selected; (2) the optimal picking sequence and corresponding tomato picking maturity; (3) which order is delivered by which type of vehicle to the consumers and the optimal served sequence, with the objective of minimizing the total cost, including distribution cost and discipline cost.

Table 2. Notations.

\begin{tabular}{cl}
\hline Sets & \\
\hline$M$ & Type of vehicles indexed with $m, m \in M$ \\
\hline$N$ & Consumers indexed with $d, d \in N$ \\
\hline$V$ & $\begin{array}{l}\text { The tomato production base and consumer nodes indexed with } i . V=N \cup\{0\}, \\
\text { indicates the tomato production base }\end{array}$ \\
\hline$K$ & Number of vehicle $m$ indexed with $k, k \in K$ \\
\hline Parameters & \\
\hline$F_{k}^{m}$ & The fixed cost of vehicle $k$ of $m$ \\
\hline$c_{i j}^{m}$ & Unit shipping cost of vehicle $m$ from customer $i$ to customer $j$ \\
\hline$v_{m}$ & Transportation speed of vehicle $m$ \\
\hline$\alpha$ & Unit discipline cost for vehicles arriving earlier than time windows \\
\hline$\beta$ & Unit discipline cost for vehicles arriving later than time windows \\
\hline$\left(E T_{i}, L T_{i}\right)$ & Time windows requested by customer $i$ \\
\hline$R$ & Optimal tomato firmness \\
\hline$r_{i}$ & Tomato picking firmness of customer $i$ \\
\hline$q_{i}$ & The demand of customer $i$ \\
\hline$Q_{m}$ & Capacity of vehicle $m$ \\
\hline$n_{m}$ & Total number of vehicle $m$ \\
\hline
\end{tabular}


Table 2. Cont.

\begin{tabular}{cl}
\hline Decision variables & \\
\hline$d_{i j}$ & Distance between customer $i$ and customer $j$ \\
\hline$\left(T_{p s i k}^{m}, T_{\text {peik }}^{m}\right)$ & $\begin{array}{l}\text { Tomato picking period of customer } i . T_{p s i k}^{m} \text { denotes the starting time of picking } \\
\text { products of customer } i \text { with vehicle } k \text { of } m ; T_{\text {peik }}^{m} \text { denotes the ending time of } \\
\text { picking products of customer } i \text { with vehicle } k \text { of } m\end{array}$ \\
\hline$\left.T_{s k}^{m}, T_{e i k}^{m}\right)$ & $\begin{array}{l}\text { Transported period of vehicle } k \text { of } m \text { delivering products from the base to } \\
\text { customer } i . T_{\text {sk }}^{m} \text { denotes the time that vehicle } k \text { of } m \text { starts delivery; } T_{\text {eik }}^{m} \text { denotes } \\
\text { the time that vehicle } k \text { of } m \text { arrives at node } i\end{array}$ \\
\hline$T_{p E k}^{m}$ & The end time that vehicle $k$ of $m$ finishes all picking orders \\
\hline$L_{i j}$ & Arc length from customer $i$ to customer $j$ \\
\hline$t_{i j}^{m}$ & Shipping time from customer $i$ to customer $j$ \\
\hline$x_{i j k}^{m}$ & $\{1$, Vehicle $k$ of $m$ delivers to customer $i ; 0$, if not $\}$ \\
\hline & $\{1$, Vehicle $k$ of $m$ delivers from customer $i$ to customer $j ; 0$, if not $\}$ \\
\hline
\end{tabular}

\subsection{Objective Function}

In the integrated tomato picking and distribution model, we take two different objectives into consideration, i.e., the distribution cost and the discipline cost generated by time windows.

\section{(1) Distribution Cost}

Let $F_{k}^{m}$ denote the fixed cost of vehicle $k$ of $m$, and $x_{o j k}^{m}$ is a binary decision variable, taking the value of 1 if vehicle $k$ of $m$ delivers products from the tomato production base to customer $j$, and 0 otherwise. Let $c_{i j}^{m}$ denote the unit shipping cost from node $i$ (tomato production base or customers) to node $j$ (tomato production base or customers) using vehicle $m . d_{i j}$ is the distance between node $i$ and node $j . x_{i j k}^{m}$ is a binary decision variable, taking the value of 1 if vehicle $k$ of $m$ delivers from customer $i$ to customer $j$, and 0 otherwise. Distribution cost can be calculated as

$$
{ }_{o b j} 1=\sum_{m=1}^{m} \sum_{k=1}^{k} F_{k}^{m} \sum_{j=1}^{n} x_{o j k}^{m}+\sum_{i=0}^{n} \sum_{j=0}^{n} \sum_{m=1}^{m} \sum_{k=1}^{k} c_{i j}^{m} d_{i j} x_{i j k}^{m}
$$

\section{(2) Discipline Cost}

We consider time windows in this study to satisfy customer demand. Discipline cost can be divided into two parts. One is the part where vehicles arrive before time windows and the other is after time windows. Both of the two parts have different impacts on customers, hence the use of two parameters, $\alpha$ and $\beta$, to denote the different extent of the time. The calculation is given in

$$
o b j 2=\alpha \sum_{i=1}^{n} \max \left(E T_{i}-T_{e i k^{\prime}}^{m} 0\right)+\beta \sum_{i=1}^{n} \max \left(T_{\text {eik }}^{m}-L T_{i}, 0\right) .
$$

\subsection{Constraints}

We formulated a multiple-vehicle routing problem with time windows, and the constraints are given as follows:

$$
\begin{gathered}
\sum_{i=0}^{n} q_{i} y_{i k}^{m} \leq Q_{m}, k \in K, m \in M \\
\sum_{k=1}^{k} \sum_{j=1}^{n} x_{o j k}^{m} \leq n_{m}, m \in M \\
\sum_{i=0}^{n} \sum_{j=0}^{n} x_{i j k}^{m} \leq|S|-1, k \in K, m \in M, S \subset V \backslash\{0\}
\end{gathered}
$$




$$
\begin{aligned}
& \sum_{j=0}^{n} x_{0 j k}^{m}=\sum_{i=0}^{n} x_{i o k}^{m} \leq 1, k \in K, m \in M \\
& \sum_{\substack{i=0 \\
i \neq p}}^{n} x_{i p k}^{m}-\sum_{\substack{j=0 \\
j \neq p}}^{n} x_{p j k}^{m}=0, k \in K, m \in M \\
& \sum_{i=0}^{n} x_{i j k}^{m}=y_{j k^{\prime}}^{m}, j \in V, k \in K, m \in M \\
& i \neq j \\
& \sum_{j=0}^{n} x_{i j k}^{m}=y_{i k}^{m}, i \in V, k \in K, m \in M \\
& i \neq j \\
& \sum_{m=1}^{m} \sum_{k=1}^{k} y_{i k}^{m}=1, i \in V, m \in M \\
& \sum_{m=1}^{m} \sum_{k=1}^{k} q_{j} x_{i j k}^{m} \leq L_{i j} \leq \sum_{m=1}^{m} \sum_{k=1}^{k}\left(Q_{m}-q_{i}\right) x_{i j k}^{m}, i, j \in V \\
& T_{e i k}^{m}+t_{i j} x_{i j k}^{m}-M\left(1-x_{i j k}^{m}\right) \leq T_{e j k}^{m}, i, j \in V, k \in K, m \in M \\
& T_{p E k}^{m}=\max \left\{T_{\text {peik }}^{m}\right\}, k \in K, m \in M \\
& T_{p E k}^{m}+M\left(1-x_{o i k}^{m}\right) \leq T_{s k}^{m}, i \in V, k \in K, m \in M \\
& T_{e j k}^{m}=\sum_{i=1}^{n} x_{i j k}^{m}\left(T_{e i k}^{m}+t_{i j}\right)+T_{s k}^{m}, i, j \in V, k \in K, m \in M \\
& i \neq j \\
& R^{-1}\left(r_{i}\right)+T_{e i k}^{m}-T_{p e i}=R^{-1}(R) \\
& r_{i} \in\{5,6,7,8,9,10\} \\
& x_{i j k^{\prime}}^{m}, x_{i k}^{m} \in\{0,1\} \\
& d_{i j}, T_{p s i k^{\prime}}^{m} T_{\text {peik }}^{m}, T_{s k^{\prime}}^{m}, T_{e i k^{\prime}}^{m} T_{p E k^{\prime}}^{m} t_{i j} \geq 0
\end{aligned}
$$

Constraint (4) limits the load of each vehicle, which cannot exceed its rated capacity. Constraint (5) indicates that the number of vehicles selected cannot exceed the capacity of the base. Constraint (6) denotes that subloops are eliminated. Constraint (7) limits each vehicle departing and returning to the tomato production base to, at most, one time. Constraint (8) points out flow conservation, which means once the vehicles arrive at the required location and finish the service, it will leave immediately. Constraints (9) and (10) indicate that there is only one vehicle on each route. Constraint (11) denotes that each customer is served only by one vehicle and only once. Constraint (12) constrains the arc traffic of vehicles. Constraint (13) points out the time relationship between one node and the subsequent nodes. Constraint (13) notes that the time when the vehicle reaches the next node is later than the time when it reaches the current node, and $M$ is a positive number with a large value. Constraint (14) indicates the final time when all the tomato orders that the vehicle needs to distribute are picked. Constraint (15) denotes that the delivery can only start after finishing all the picking. Constraint (16) calculates the distribution time to the customer, ignoring service time and order processing time. Constraint (17) indicates that the tomato maturity delivered to customers is optimal. Constraints (18)-(20) define the conditions on the decision variables. 


\subsection{Programming Model}

According to the discussion in Sections 3.2 and 3.3, the total objective function is to minimize obj 1 and $o b j 2$ by subjecting them to Constraints (4)-(20). The integrated tomato picking and distribution scheduling model can be expressed as

$$
\begin{aligned}
& \min o b j 1+o b j 2 \\
& \text { s.t. }(4) \sim(20)
\end{aligned}
$$

Note that Model (21) is a multiple-vehicle routing problem with time windows (m-VRPTW), and it has proven to be an NP-hard problem [27]. If the tomato production base only has one type of vehicle, Model (21) is equivalent to the VRPTW problem. If Constraints (13)-(18) are removed and $E T_{i}=0, L T_{i}=M$, and only one type of vehicle is available, Model (21) is equivalent to the VRP problem.

\section{Model Solution Based on Sweep-Adaptive Genetic Algorithm}

The genetic algorithm (GA), as a heuristic algorithm, is often used to solve VRP problems [28-30]. It simulates the evolution mechanism in nature to search for the optimal solution. GA starts from the initial population and continuously changes the population chromosomes by mutual iteration to seek the approximate optimal solution [31]. It has some disadvantages, such as high time complexity and slow convergence speed. However, the sweep algorithm can divide problems into several parts for parallel operation, which effectively improves the search speed. The self-adaptive algorithm plays an important role in improving convergence accuracy and accelerating the convergence speed. Based on the advantages of the above algorithms, this study proposes a sweep-adaptive genetic algorithm to solve the integrated tomato picking and distribution model.

The sweep-adaptive genetic algorithm (S-AGA) is a hybrid heuristic algorithm based on the genetic algorithm. This study formulates corresponding criteria and forms multiple effective initial solutions according to constraints. Then, the optimal solution is reached through selection, adaptive crossover, variation, and termination conditions, minimizing the total distribution and discipline costs. The pseudocode of the algorithm is shown in Algorithm 1 below.

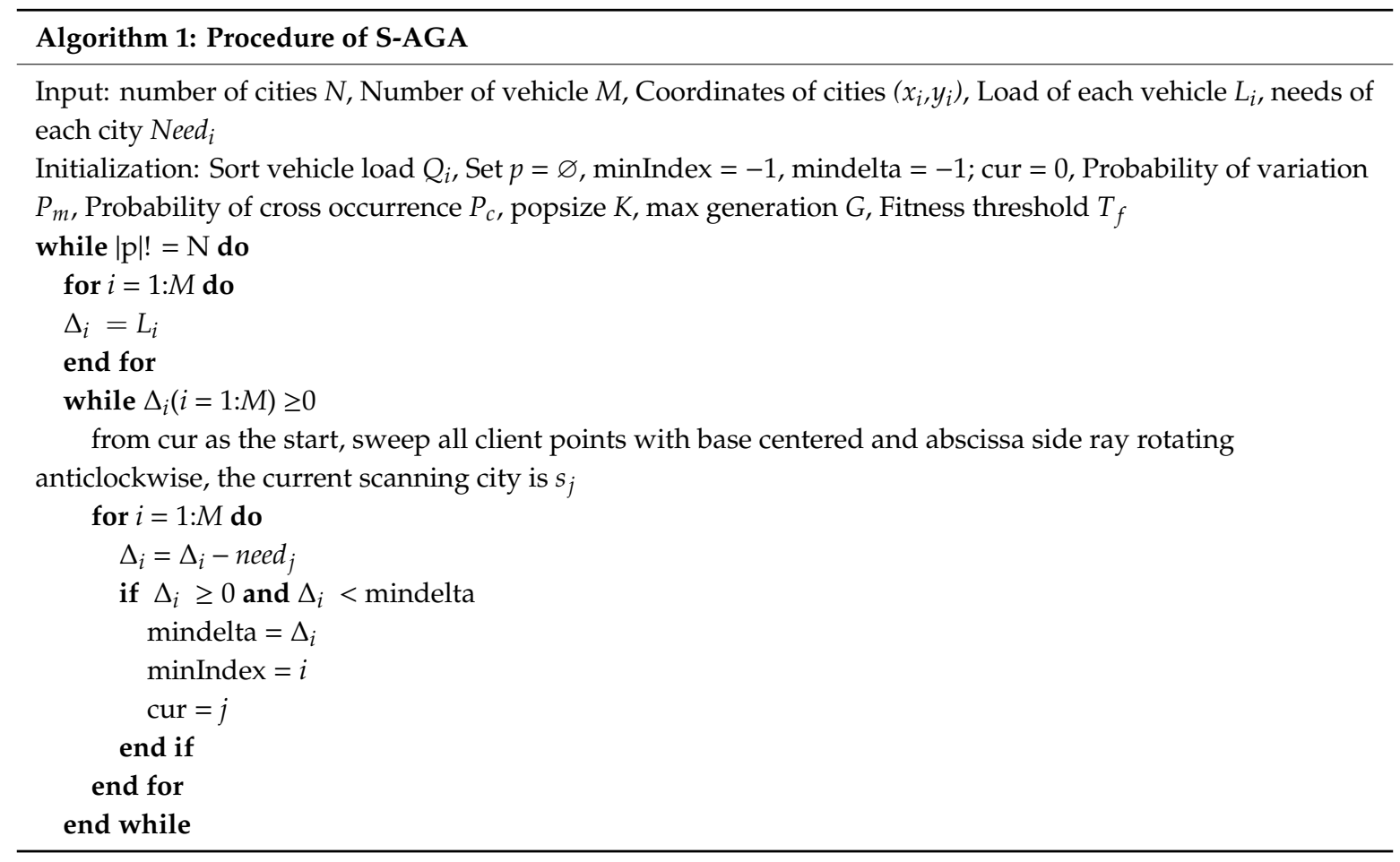




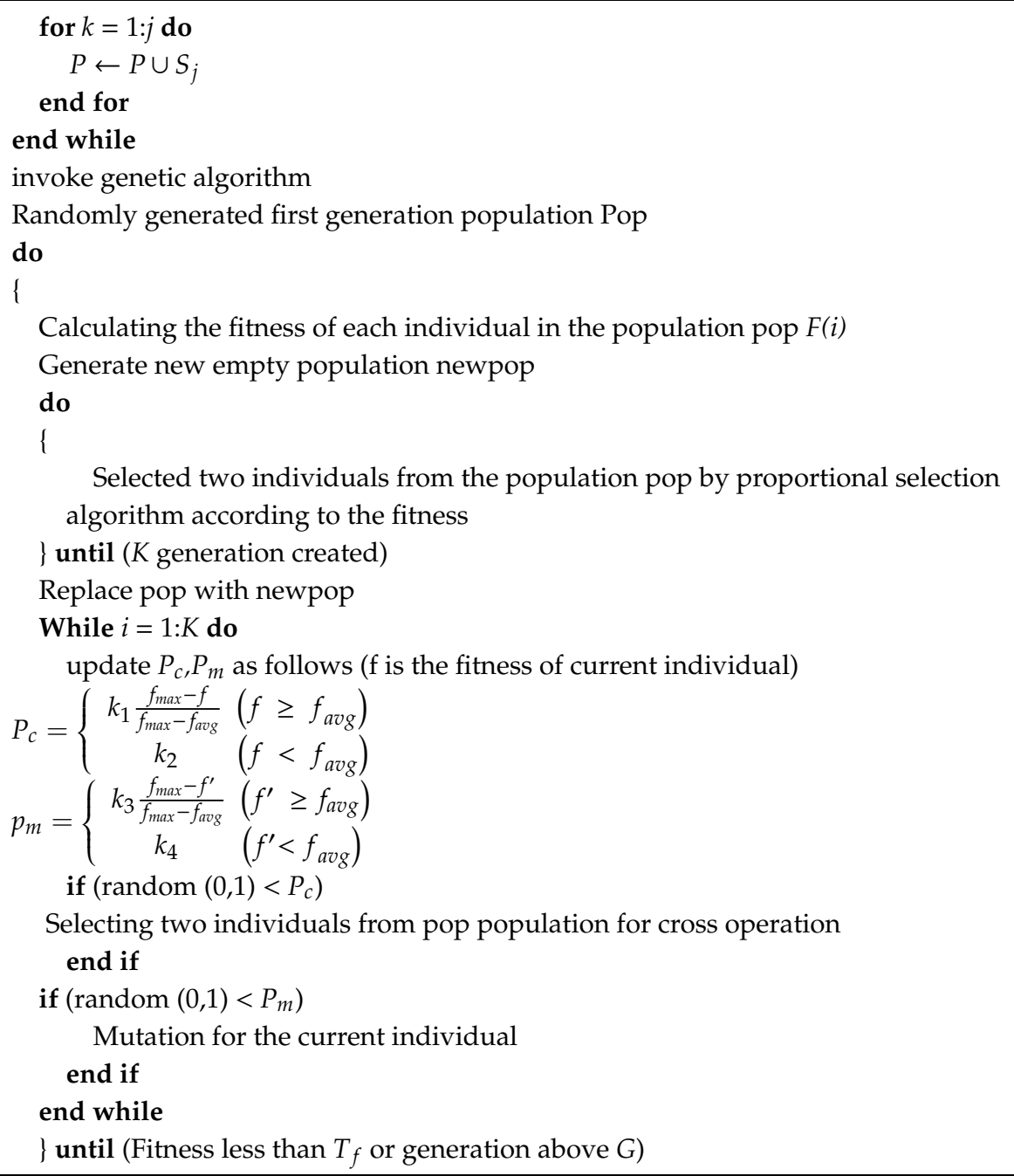

\subsection{Code and Population Initialization}

The feasible solution is expressed by the chromosome in GA. Additionlly, we use the integer coding method to encode the chromosome; 0 represents the tomato production base, and the remaining nodes are added with 1 based on the original serial number. For example, chromosome $S=174,216,351$. As shown in chromosome $S$, there are two routes, including Customers 7, 4, and 2 served in Route 1 and Customers 6, 3, and 5 served in Route 2. They are all served successively.

The initial population is the origin of evolution, which has an impact on the operation performance of GA so that the population size should be carefully selected. In this paper, we divide the population into several parallel subpopulations. Each subpopulation represents the feasible solution of each vehicle, being a part of the optimal solution of the optimization model. There are many ways of initialization, such as chaotic initialization and random initialization. Random initialization is selected in this study.

\subsection{Constraint Treatment}

Traditional GA has a high time-complexity. It is not suitable for large-scale studies. The sweep algorithm is used in this study to reduce the difficulty of model solving, preprocessing data, and meeting the constraints of load and number of vehicles via grouping first and processing later. It is shown in Algorithm 2 from Step 1 to Step 5, translating the VRP problem into several simple TSP problems. 


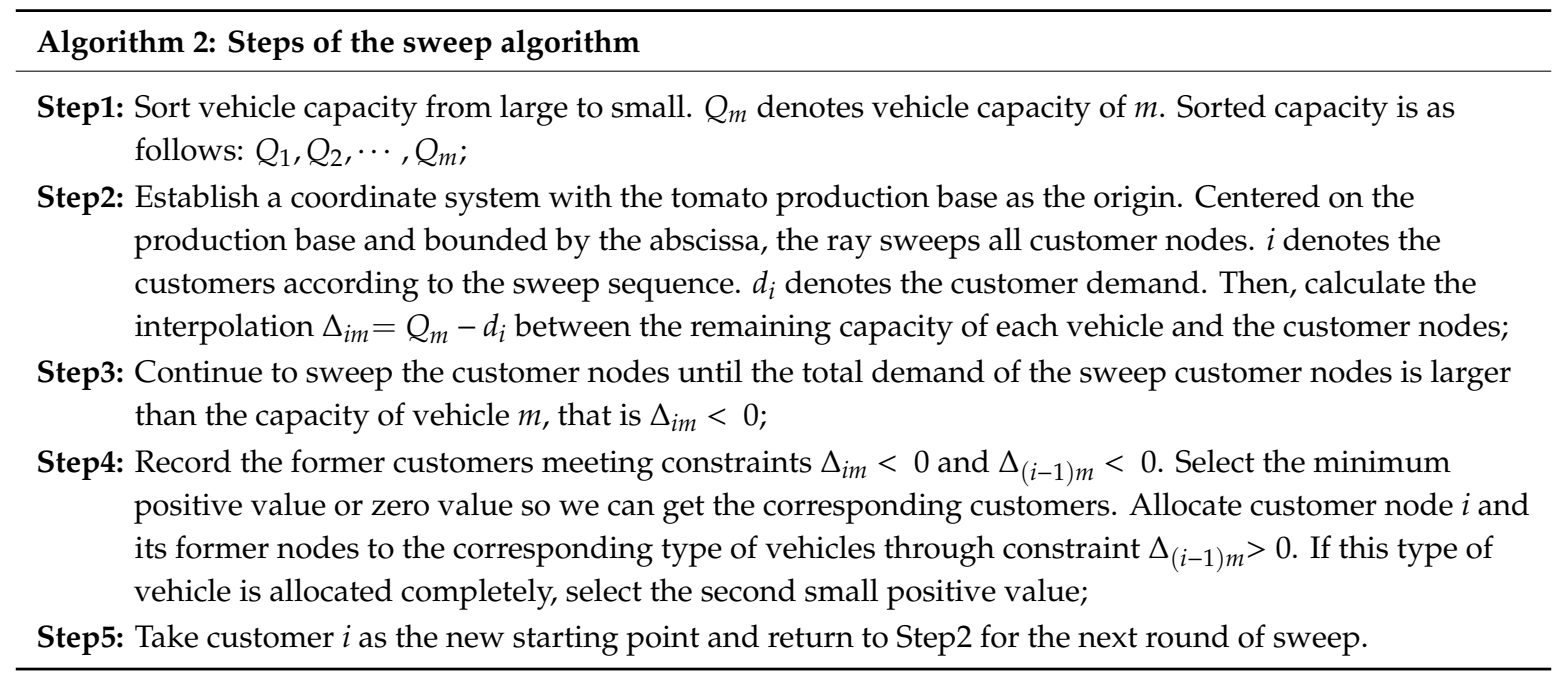

\subsection{Fitness and Selection}

Fitness can be used to evaluate the quality of a chromosome. Chromosomes of high quality are easy to survive and exist on a large scale, while those of poor quality will exist on a small scale or even be extinct. The objective function is to minimize the total cost. To get the optimal solution, we use the reciprocal of the objective function to denote the fitness, as shown in Equation (23). $Z_{i}$ indicates the objective function value of chromosome $i$.

$$
\operatorname{Fit}(i)=\frac{1}{Z_{i}}
$$

The selection calculation is to determine the probability of being selected according to the size of the fitness. Higher fitness will be better selected. The next generation of surplus individuals is calculated by the proportional selection method.

\subsection{Crossover and Mutation}

Crossover and mutation operation can generate new individuals and increase population diversity. In this study, a sequential crossover is used, and a self-adaptive crossover is added to improve population diversity [32], as shown in Equation (24). The crossover operation of this paper is order crossover. For example, Chromosome A = 28410517369 or Chromosome B = 56711028394 . First, select a gene sequence with the same position in Chromosomes A and B randomly, such as the 3 to 6 genes in Chromosomes A (__ $\left.41051_{-{ }_{-}}\right)$and B (__ $\left.71102_{-{ }_{-}}\right)$. Then, keep the proposed gene sequence unchanged, exchange the remaining gene sequences of Chromosomes $\mathrm{A}$ and $\mathrm{B}$ in the original order, that is, the new Chromosomes A1 = $\underline{6} \underline{7} 41051 \underline{2} \underline{8} \underline{3} \underline{9}$ and B1 = $\underline{8} \underline{4} 71102 \underline{5} \underline{3} \underline{6} \underline{9}$.

$$
P_{c}=\left\{\begin{array}{cl}
k_{1} \frac{f_{\max }-f}{f_{\max }-f_{\text {avg }}} & \left(f \geq f_{\text {avg }}\right) \\
k_{2} & \left(f<f_{\text {avg }}\right)
\end{array}\right.
$$

Mutation operation uses self-adaptive mutation to make variations more flexible [30], as shown in Equation (25). Mutation operation uses exchange mutation. Exchange any two genes' positions on a chromosome. For example, Chromosome $C=173946582$ 10; then, exchange two genes' positions randomly, such as " 3 " and "10". The new chromosome C1 = $17 \underline{10} 945582 \underline{3}$.

$$
p_{m}=\left\{\begin{array}{cl}
k_{3} \frac{f_{\max }-f^{\prime}}{f_{\max }-f_{\text {avg }}} & \left(f^{\prime} \geq f_{\text {avg }}\right) \\
k_{4} & \left(f^{\prime}<f_{\text {avg }}\right)
\end{array}\right.
$$


where $f$ and $f^{\prime}$ denote the larger fitness of two individuals by crossover or mutation operations; $k_{1}, k_{2}$, $k_{3}, k_{4}$ are constants; $f_{\max }$ indicates the largest fitness of the population; $f_{\text {avg }}$ denotes the average fitness of the population.

\subsection{Termination Condition}

The termination condition of this paper achieves a predetermined set of evolutionary generations when iterations reach 1000 or the fitness value between successive generations is less than the threshold value. Otherwise, the above steps will be repeated until the termination conditions are met. When evolution is stopped, select the chromosome with the highest fitness in each subpopulation as the corresponding optimal scheme. All the schemes are combined together to get the overall scheme design of the problem. The overall process is shown in Figure 3.

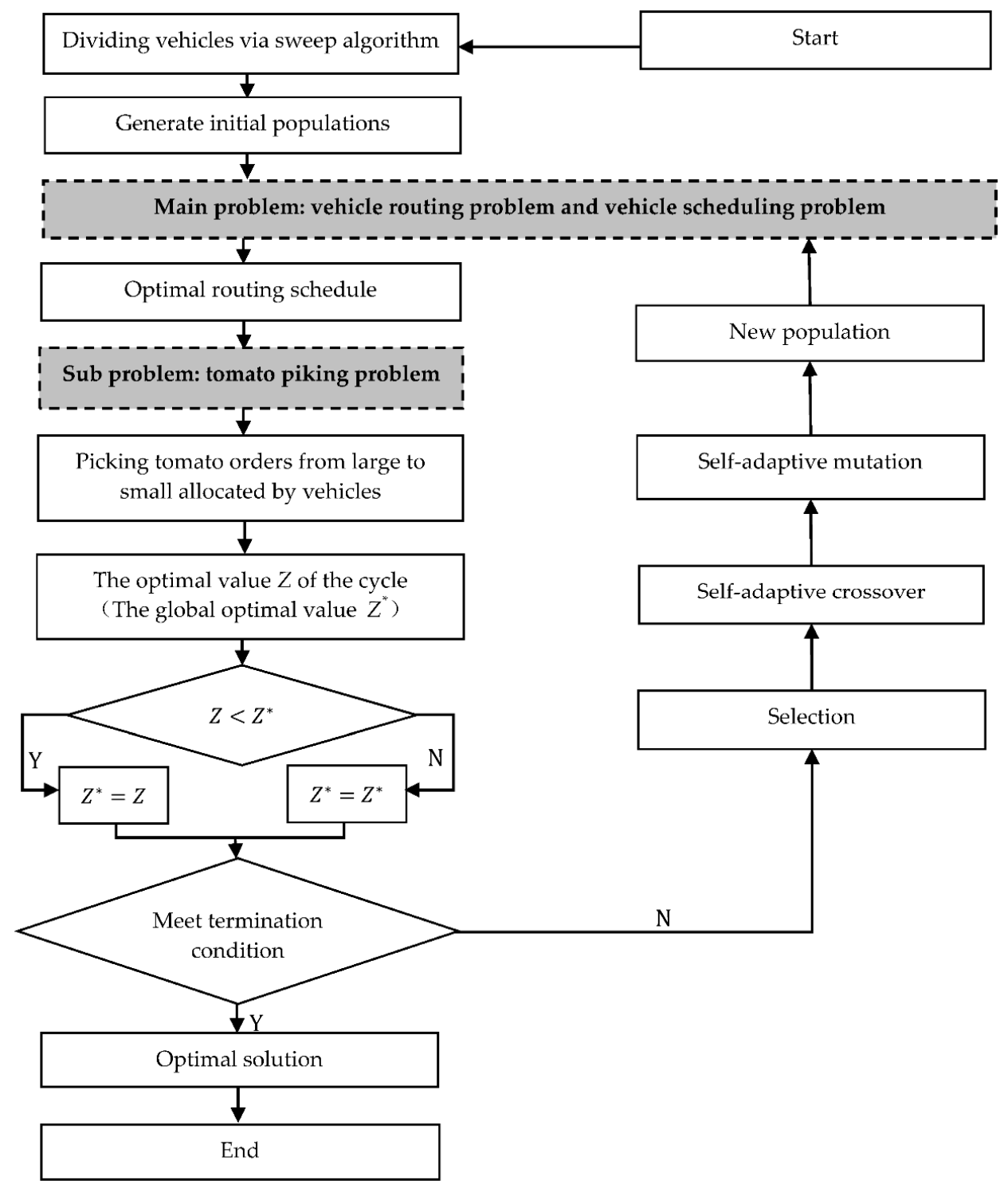

Figure 3. Flow scheme of the sweep-adaptive genetic algorithm (S-AGA).

\section{Numerical Experiments and Simulation Analysis}

To verify the effectiveness of the model, this study used S-AGA and traditional GA for a simulation operation aimed at different scales and compared the results. In this section, we adopt MATLAB R2017b as an MINLP solver to solve the integrated tomato picking and distribution model. In Section 5.1, 
a small-scale numerical experiment with one tomato production base and 20 customers is carried out to prove the efficiency and feasibility of the proposed model. In Section 5.2, we address 11 different large-scale numerical experiments to verify the superiority of S-AGA compared with traditional GA.

\subsection{Small-Scale Example Setting}

In this example, the tomato supply chain consists of a tomato production base ( 0 ) and 20 customers (1-20). The customer locations, demands, requested time windows, and the related information of the tomato production base are all known.

The tomato production base has two types of vehicles. The tomato picking speed of the production base is $1 \mathrm{t} / \mathrm{h}$, and the change of tomato maturity after picking is consistent with the proposed tomato maturity model. According to practical experience, the optimal tomato maturity is 9 , corresponding to firmness $29 \mathrm{~N}$. Considering daily life, customers are more likely to receive the agriproducts early. Therefore, we set $\alpha=0$ and $\beta=20 \mathrm{CNY} / \mathrm{h}$. At the same time, we set related parameters of algorithm S-AGA, including the initial population of 50, maximum iterations of 500, and self-adaptive crossover and mutation parameters $k_{1}=1.0, k_{2}=0.5, k_{3}=0.8, k_{4}=0.5$. Table 3 demonstrates the details of 20 orders. Table 4 gives the vehicle information.

Table 3. Customer information.

\begin{tabular}{ccccc}
\hline \multirow{2}{*}{ Customer Nodes } & Demand (t) & Coordinates $\mathbf{( k m )}$ & \multicolumn{2}{c}{ Time Windows } \\
\cline { 4 - 5 } & & & $\boldsymbol{E} \boldsymbol{T}_{\boldsymbol{i}} \mathbf{( h )}$ & $\boldsymbol{L T}_{\boldsymbol{i}} \mathbf{( h )}$ \\
\hline 0 & 0 & $(0,0)$ & 0 & 0 \\
1 & 0.015 & $(50,555)$ & 30 & 52 \\
2 & 0.7 & $(255,80)$ & 0 & 22 \\
3 & 0.005 & $(382,101)$ & 16 & 35 \\
4 & 0.005 & $(624,325)$ & 24 & 58 \\
5 & 0.01 & $(475,254)$ & 18 & 74 \\
6 & 2.3 & $(461,431)$ & 13 & 87 \\
7 & 0.015 & $(389,766)$ & 45 & 92 \\
8 & 0.01 & $(310,350)$ & 8 & 61 \\
9 & 1.2 & $(370,428)$ & 0 & 89 \\
10 & 0.005 & $(520,690)$ & 4 & 86 \\
11 & 0.010 & $(193,439)$ & 9 & 34 \\
12 & 0.015 & $(231,343)$ & 16 & 43 \\
13 & 0.9 & $(165,264)$ & 21 & 29 \\
14 & 0.01 & $(206,251)$ & 17 & 35 \\
15 & 0.01 & $(140,577)$ & 14 & 92 \\
16 & 1.1 & $(105,365)$ & 10 & 74 \\
17 & 0.005 & $(13,353)$ & 5 & 33 \\
18 & 0.01 & $(74,510)$ & 17 & 72 \\
19 & 0.015 & $(375,739)$ & 15 & 52 \\
20 & 1.4 & $(446,90)$ & 21 & 52 \\
\hline
\end{tabular}

Table 4. Vehicle information.

\begin{tabular}{cccccc}
\hline Type & Speed(km/h) & Load(t) & Number & Vehicle Cost (CNY) & Unit Shipping Cost (CNY/km) \\
\hline A & 30 & 4 & 5 & 200 & 2 \\
B & 30 & 6 & 3 & 230 & 2.2 \\
\hline
\end{tabular}

\subsection{Numerical Results and Management Significance}

The basic numerical experiment is tested 10 times, and the optimal scheduling of the tomato supply chain is reported in Table 5, including vehicle selection, tomato picking sequence, picking maturity, and firmness, as well as the optimal route schedule. Tomato picking maturity can be obtained by the corresponding table of firmness and maturity shown in Figure 1. The firmness interval $(31,35)$ 
corresponds to Maturity 8 , while the firmness interval $(27,31)$ corresponds to Maturity 9 . We take the order of the table column as the picking order. The 20 customers are represented on the distance axis in the form of a hollow circular in Figure 4. Blue lines denote the route of Vehicle A, while red lines denote the route of Vehicle B, depicted in Figure 4a. The evolution graph of S-AGA is depicted in Figure $4 \mathrm{~b}$. It can be seen that the proposed model can provide a complete scheme of the tomato supply chain, improving the overall operational efficiency of logistics enterprises and the product quality service.

Table 5. The optimal scheduling of the tomato picking supply chain.

\begin{tabular}{ccc}
\hline Scheme & Vehicle B (6t) & Vehicle A (4t) \\
\hline \multirow{2}{*}{ Picking sequence } & $\begin{array}{c}2.3 \mathrm{t} \rightarrow 1.4 \mathrm{t} \rightarrow 1.2 \mathrm{t} \rightarrow 0.7 \mathrm{t} \rightarrow 0.015 \mathrm{t} \rightarrow 0.01 \mathrm{t} \rightarrow \\
0.01 \mathrm{t} \rightarrow 0.01 \mathrm{t} \rightarrow 0.005 \mathrm{t} \rightarrow 0.005 \mathrm{t} \rightarrow 0.005 \mathrm{t}\end{array}$ & $\begin{array}{c}1.1 \mathrm{t} \rightarrow 0.9 \mathrm{t} \rightarrow 0.015 \mathrm{t} \rightarrow 0.015 \mathrm{t} \rightarrow 0.015 \mathrm{t} \rightarrow \\
0.01 \mathrm{t} \rightarrow 0.01 \mathrm{t} \rightarrow 0.01 \mathrm{t} \rightarrow 0.005 \mathrm{t}\end{array}$ \\
\hline \multirow{2}{*}{ Picking firmness (N) } & $\begin{array}{c}32.5 \rightarrow 29.3 \rightarrow 32.2 \rightarrow 28.9 \rightarrow 30.0 \rightarrow 30.5 \rightarrow \\
30.0 \rightarrow 29.6 \rightarrow 29.0 \rightarrow 30.6 \rightarrow 31.4\end{array}$ & $\begin{array}{c}31.8 \rightarrow 28.9 \rightarrow 30.8 \rightarrow 30.7 \rightarrow 30.0 \rightarrow \\
29.6 \rightarrow 30.0 \rightarrow 29.7 \rightarrow 29.1\end{array}$ \\
\hline Picking maturity & $8 \rightarrow 9 \rightarrow 8 \rightarrow 9 \rightarrow 9 \rightarrow 9 \rightarrow 9 \rightarrow 9 \rightarrow 9 \rightarrow 9 \rightarrow 8$ & $8 \rightarrow 9 \rightarrow 9 \rightarrow 9 \rightarrow 9 \rightarrow 9 \rightarrow 9 \rightarrow 9 \rightarrow 9$ \\
\hline \multirow{2}{*}{ Route scheduling } & Base $\rightarrow i_{2} \rightarrow i_{3} \rightarrow i_{20} \rightarrow i_{14} \rightarrow i_{12} \rightarrow$ & Base $\rightarrow i_{13} \rightarrow i_{17} \rightarrow i_{11} \rightarrow i_{18} \rightarrow i_{1} \rightarrow$ \\
$i_{8} \rightarrow i_{5} \rightarrow i_{4} \rightarrow i_{10} \rightarrow i_{9} \rightarrow i_{6} \rightarrow$ Base & $i_{15} \rightarrow i_{19} \rightarrow i_{7} \rightarrow i_{16} \rightarrow$ Base \\
\hline Cost (CNY) & 6134.98 & 4892.05 \\
\hline
\end{tabular}

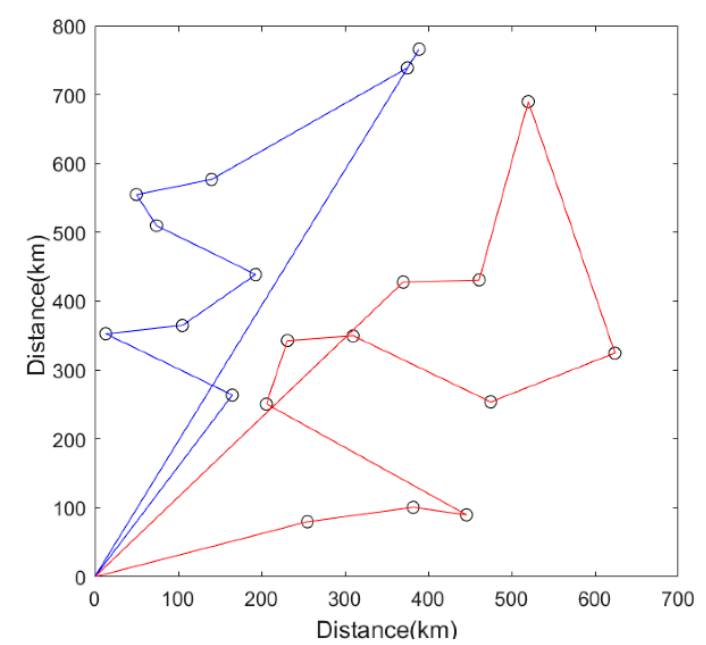

(a)

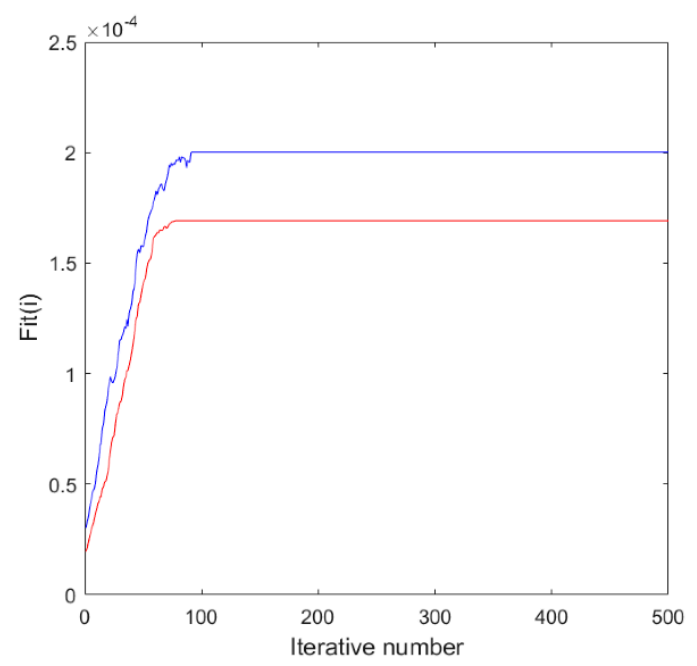

(b)

Figure 4. Optimal routes and evolution graph. (a) Optimal route of two vehicles; (b) evolution graph of S-AGA.

In Figure 4a, we obviously find that the route of Vehicle B is not the shortest because we should consider time windows of customers and weigh the cost of logistics against the discipline cost of tomatoes not being delivered within the request time windows in real life. If we pay more logistics costs than discipline costs, we may ignore customer satisfaction to minimize the whole cost. However, if we pay more discipline costs compared with logistics costs, enterprises would like to take the longer way to meet time windows and customer satisfaction. In fact, in order to minimize the overall tomato supply chain cost, we should consider a reasonable number and type of vehicles and routes.

We ignore the time of tomato picking and only consider the deterioration in the process of transportation. The new optimal scheme of the tomato supply chain is reported in Table 6, and new optimal routes and the evolution graph are described in Figure 5. It can be seen that part of the firmness and maturity of tomatoes change when they are delivered. 
Table 6. The new optimal schedule, neglecting the time of tomato picking.

\begin{tabular}{ccc}
\hline Scheme & Truck B (6t) & Truck A (4t) \\
\hline \multirow{2}{*}{ Picking firmness (N) } & $\begin{array}{c}31.9 \rightarrow 29.0 \rightarrow 32 \rightarrow 28.7 \rightarrow 29.8 \rightarrow 30.2 \rightarrow \\
30.0 \rightarrow 29.6 \rightarrow 29.0 \rightarrow 30.6 \rightarrow 31.4\end{array}$ & $\begin{array}{c}29.2 \rightarrow 28.7 \rightarrow 30.6 \rightarrow 30.6 \rightarrow 29.8 \rightarrow \\
29.5 \rightarrow 30.0 \rightarrow 29.7 \rightarrow 29.1\end{array}$ \\
\hline Picking maturity & $8 \rightarrow 9 \rightarrow 8 \rightarrow 9 \rightarrow 9 \rightarrow 9 \rightarrow 9 \rightarrow 9 \rightarrow 9 \rightarrow 9 \rightarrow 8$ & $8 \rightarrow 9 \rightarrow 9 \rightarrow 9 \rightarrow 9 \rightarrow 9 \rightarrow 9 \rightarrow 9 \rightarrow 9$ \\
\hline \multirow{2}{*}{ Route schedule } & $\begin{array}{l}\text { Base } \rightarrow i_{2} \rightarrow i_{3} \rightarrow i_{20} \rightarrow i_{14} \rightarrow i_{12} \rightarrow \\
i_{8} \rightarrow i_{5} \rightarrow i_{4} \rightarrow i_{10} \rightarrow i_{6} \rightarrow i_{9} \rightarrow \text { Base }\end{array}$ & $\begin{array}{c}\text { Base } \rightarrow i_{13} \rightarrow i_{17} \rightarrow i_{16} \rightarrow i_{11} \rightarrow \\
i_{18} \rightarrow i_{15} \rightarrow i_{19} \rightarrow i_{7} \rightarrow \text { Base }\end{array}$ \\
\hline Cost (CNY) & 5911.46 & 4994.69 \\
\hline
\end{tabular}

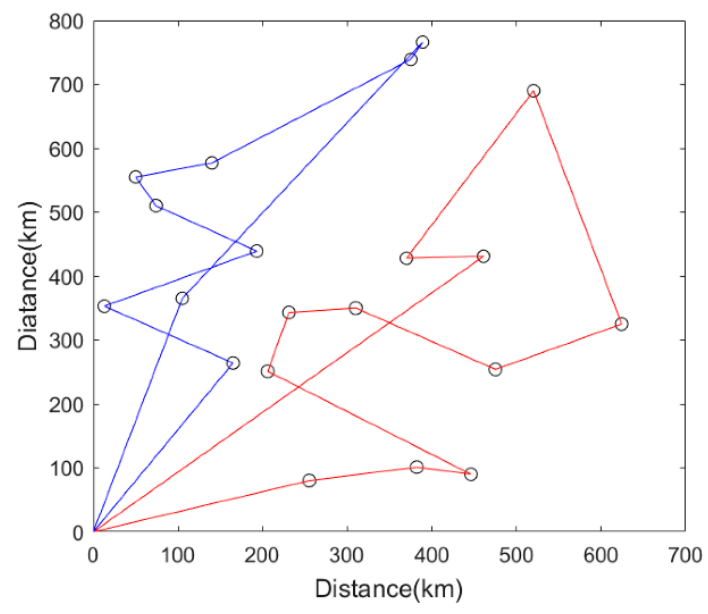

(a)

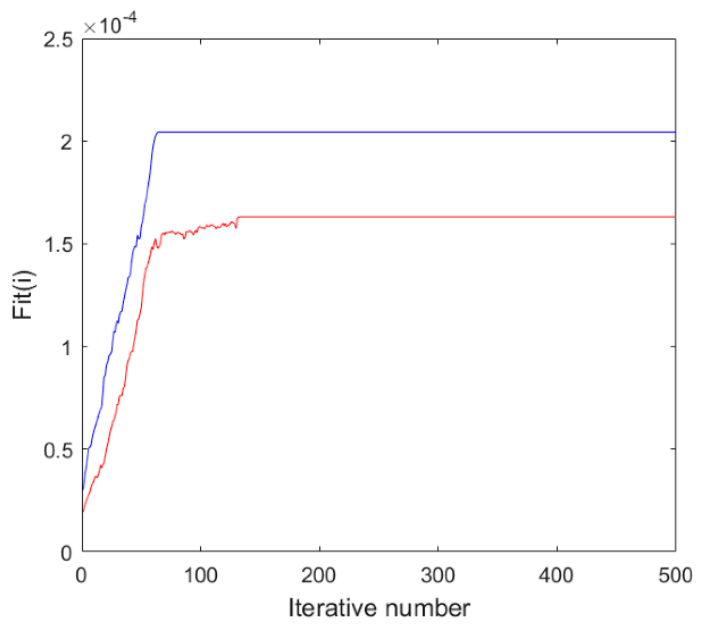

(b)

Figure 5. New optimal routes and evolution graph. (a) Optimal route of two vehicles; (b) evolution graph of S-AGA.

Comparing Table 6 with Table 5, we can find that the cost of both schemes is almost the same. The total cost is reduced by $1 \%$, while customer satisfaction based on tomato quality decreases from $100 \%$ to $95 \%$. The example in this section is on a small scale; the change of tomato maturity is not significant for short-distance distribution. However, in modern society, with convenient transportation, delivery areas of suppliers are larger. Moreover, for some agricultural products like juicy peach, whose maturity changes faster, the quality change of agricultural products will be more obvious, and the superiority of this model will be further highlighted.

\subsection{Large-Scale Example Setting and Performance Analysis of Algorithm}

In this section, we use 11 different large-scale numerical experiments to demonstrate the advantage and efficiency of S-AGA. Customer size increases from 50 to 1000. The computational performance results of S-AGA and GA are reported via CPU time and iteration number.

We generate $N$ customers randomly. Furthermore, among them, $70 \%$ are online customers, while $30 \%$ are offline customers. The online customer demand $D_{i}$ follows the uniform distribution $\mathrm{U}(0.005,0.015)$, while the offline customer demand $D_{i}$ follows the uniform distribution $\mathrm{U}(0.6,2)$. The earliest arrival time $E T_{i}$ is generated by $\mathrm{U}(0,24)$, and the latest arrival time $L T_{i}$ is generated by $E T_{i}+\mathrm{U}(15,50)$.

We reported the computational results of GA and S-AGA in Table 7. It can be seen that with the increase in customer scale, GA and S-AGA show an increasing trend in CPU time and iterations. However, the time gap of S-AGA is much faster than that of GA, which is more than $90 \%$. Additionally, S-AGA shows more advantages of iterations, reducing more than $50 \%$ compared with GA. 
Table 7. Numerical results of large-scale cases.

\begin{tabular}{cccccc}
\hline \multirow{2}{*}{ Case } & \multirow{2}{*}{ Variable Sizes } & \multicolumn{2}{c}{ S-AGA } & \multicolumn{2}{c}{ GA } \\
\cline { 3 - 6 } & & CPU (s) & Iterations & CPU (s) & Iterations \\
\hline 1 & 50 & 21.37 & 223 & 297.21 & 511 \\
2 & 100 & 49.80 & 368 & 1201.85 & 876 \\
3 & 200 & 130.83 & 294 & 1294.76 & 953 \\
4 & 300 & 179.93 & 381 & 2203.74 & 976 \\
5 & 400 & 210.93 & 590 & 3014.77 & 914 \\
6 & 500 & 281.73 & 618 & 3176.42 & 1000 \\
7 & 600 & 378.53 & 913 & 2893.61 & 1000 \\
8 & 700 & 391.08 & 415 & 4589.97 & 762 \\
9 & 800 & 456.61 & 330 & 4742.13 & 1000 \\
10 & 900 & 446.54 & 887 & 5603.74 & 1000 \\
11 & 1000 & 584.93 & 682 & 5532.17 & 1000 \\
\hline
\end{tabular}

Figure 6a demonstrates the comparison of CPU time between S-AGA and GA. It shows that S-AGA is significantly superior to GA in CPU time. Figure 6b shows the trend of iterations of S-AGA and GA. From Case 1 to Case 7, the iteration gaps become smaller, while from Case 8 to Case 9 , the iteration gaps get larger compared with Case 7. However, we find that the iterations of GA are all larger than that of S-AGA, and the largest iteration gap between S-AGA and GA is $67 \%$. Generally, the performance of S-AGA proposed in this paper is better than the traditional GA in solving integrated tomato picking and distribution. Moreover, we conclude that S-AGA has good convergence and faster convergence speed, and it is suitable for providing schemes quickly when dealing with large-scale customers.

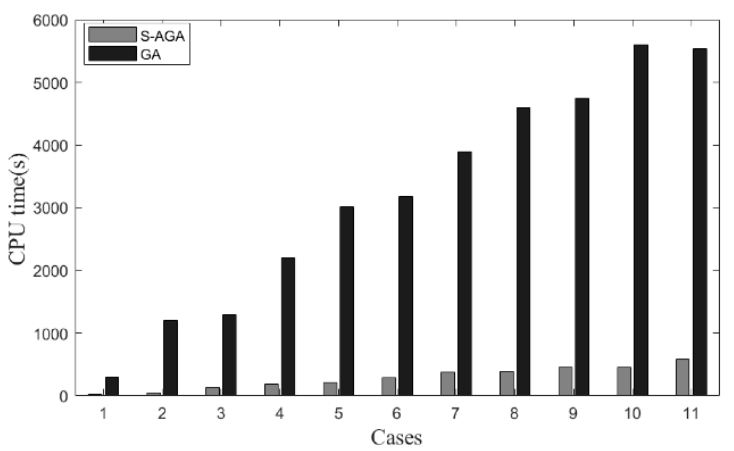

(a)

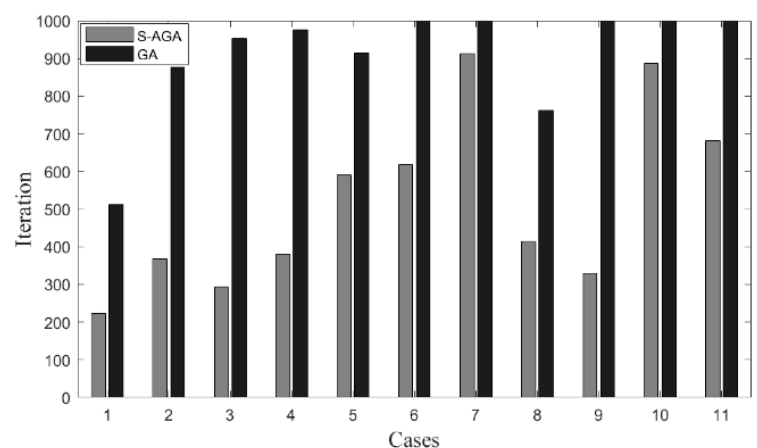

(b)

Figure 6. Comparison of computational performance between S-AGA (sweep-adaptive genetic algorithm) and GA (genetic algorithm). (a) Comparison of CPU time between S-AGA and GA; (b) comparison of iterations between S-AGA and GA.

\section{Conclusions}

This paper studied an integrated tomato picking and distribution schedule based on tomato maturity in the context of a farm-to-door model. We presented a tomato maturity model with firmness and incorporated it into the proposed integrated tomato picking and distribution model, with the objectives of minimizing the total cost in terms of obtaining optimal tomato quality and satisfaction for customers. In the proposed model, we addressed an improved algorithm, S-AGA, to solve the model and get the schedule of the tomato supply chain. In addition, numerical experiments were conducted to demonstrate the efficiency and effectiveness of S-AGA in solving the proposed model compared with traditional GA. The simulation result of a small-scale example shows that we can provide tomato quality and improve by over 5\% customer satisfaction without additional cost. Moreover, the result of a large-scale example demonstrates that both CPU time and iteration of S-AGA are superior to GA. Therefore, with the analysis of the example, the designed algorithm and proposed model established in 
this paper are reasonable. The promotion of this model is also conducive to realizing the personalized customization of customers and improving the sustainability of agriculture and logistics. Users can choose different tomato maturities to store, sell, or eat, meeting the needs of diversified customers.

There are specific areas in this study that can be researched further. This study only considered a single tomato production base; hence multiple bases could be considered as a prospect. Moreover, tomato maturity only considered one indicator, so we could consider multiple indicators to propose in the tomato maturity model. Then, another prospect could incorporate more uncertainty factors into the integrated tomato picking and distribution schedule, such as the uncertainties of transportation and the processing of tomato picking. Finally, our research is based on the context of the farm-to-door model, so another model and related factors could be taken into consideration, such as warehouse storage time. We leave these problems for future work.

Author Contributions: Conceptualization, methodology, validation, formal analysis, investigation, data curation, A.Z., B.B., Y.J., and J.H.; writing—original draft preparation, A.Z.; writing—review and editing, B.B., Y.J., and J.H. All authors have read and agreed to the published version of the manuscript.

Funding: This research was funded by the National Natural Science Foundation of China, grant number 71803084, Fundamental Research Funds for the Central Universities, grant number NAU: SKCX2020009; the Humanity and Social Science Youth Foundation of the Ministry of Education of China, grant number 17YJC630048; the Students' Research Training Program of Nanjing Agricultural University, grant number 1930A77; and Priority Academic Program Development of Jiangsu Higher Education Institutions (PAPD).

Conflicts of Interest: The authors declare no conflict of interest.

\section{References}

1. Behzadi, G.; Osullivan, M.J.; Olsen, T.L.; Zhang, A. Agribusiness supply chain risk management: A review of quantitative decision models. Omega 2018, 79, 21-42. [CrossRef]

2. Maul, F.; Sargent, S.A.; Sims, C.A.; Baldwin, E.A.; Huber, D.J. Tomato flavor and aroma quality as affected by storage temperature. J. Food Sci. 2000, 65, 1228-1237. [CrossRef]

3. Osvald, A.; Stirn, L.Z. A vehicle routing algorithm for the distribution of fresh vegetables and similar perishable food. J. Food Eng. 2008, 85, 285-295. [CrossRef]

4. Ahumada, O.; Villalobos, J.R. Application of planning models in the agri-food supply chain: A review. Eur. J. Oper. Res. 2009, 196, 1-20. [CrossRef]

5. Ahumada, O.; Villalobos, J.R.; Mason, A.N. Tactical planning of the production and distribution of fresh agricultural products under uncertainty. Agric. Syst. 2012, 112, 17-26. [CrossRef]

6. Amalia, U.; Torsten, R.; Amir, H.A. Optimisation of agricultural routing planning in field logistics with evolutionary hybrid neighbourhood search. Biosyst. Eng. 2019, 184, 166-180.

7. Javier, E.G.-L.; Marcela, C.G.-A.; Wladimir, E.S.-S.; Masly, M.R.-M. Optimizing tactical harvest planning for multiple fruit orchards using a metaheuristic modeling approach. Eur. J. Oper. Res. 2020, in press. [CrossRef]

8. Graf, P.M. Coupling of crop assignment and vehicle routing for harvest planning in agriculture. Artif. Intell. Agric. 2019, 2, 99-109.

9. Ali, O.; Verlinden, B.; Oudheusden, D.V. Infield logistics planning for crop-harvesting operations. Eng. Optimiz. 2009, 41, 183-197. [CrossRef]

10. Kamal, L.; Philip, C.J.; Barrett, W.T. Harvest logistics in agricultural systems with multiple, independent producers and no on-farm storage. Comput. Ind. Eng. 2016, 91, 129-138.

11. Liu, H.; Zhang, J.; Zhou, C. Optimal purchase and inventory retrieval policies for perishable seasonal agricultural products. Omega 2018, 79, 133-145. [CrossRef]

12. Wang, X.; Wang, M.; Ruan, J.; Zhan, H. The multi-objective optimization for perishable food distribution route considering temporal-spatial distance. Procedia Comput. Sci. 2016, 96, 1211-1220. [CrossRef]

13. Fan, J. The vehicle routing problem with simultaneous pickup and delivery based on customer satisfaction. Procedia Eng. 2011, 15, 5284-5289. [CrossRef]

14. Li, J.Y.; Fan, L.N.; Dong, D.Y. A new route optimization approach of cold chain logistics distribution based on fresh agricultural products. In Proceedings of the 30th China Conference on Control and Decision-Making, Liaoning, China, 9 June 2018; pp. 1118-1123. 
15. Wan, P.; Toudeshki, A.; Tan, H.; Ehsani, R. A methodology for fresh tomato maturity detection using computer vision. Comput. Electron. Agric. 2018, 146, 43-50. [CrossRef]

16. Mohammed, M.; Wilson, L.A.; Gomes, P.I. Postharvest sensory and physiochemical attributes of processing and nonprocessing tomato cultivars. J. Food. Qual. 1999, 22, 167-182. [CrossRef]

17. Shanna, M.; Julia, V.; Paxton, P.; Jim, G. Use of genomics tools to isolate key ripening genes and analyse fruit maturation in tomato. J. Exp. Bot. 2002, 53, 2023-2030.

18. Zhang, L.; McCarthy, M.J. Measurement and evaluation of tomato maturity using magnetic resonance imaging. Postharvest Biol. Technol. 2012, 76, 37-43. [CrossRef]

19. Wan, S.L.; Gao, Y.; Nie, J.Q.; Cai, Y.P. The changes of characters to fruit firmness of tomato during fruit ripening. China Veg. 2008, 4, 20-23.

20. Holt, C.B. Measurement of tomato firmness with a universal testing machine. J. Texture Stud. 1970, 19, 491-501. [CrossRef]

21. Patrick, L.; Marie, F.D. Measurement of tomato firmness by using a non-destructive mechanical sensor. Postharvest Biol. Technol. 1996, 8, 45-55.

22. Zsom, M.V.; Zsom, T.; Felföldi, J. In-Vivo measurement of tomato firmness. Acta Hortic. 2008, 801, 677-684. [CrossRef]

23. Liu, L.; Wang, H.; Xing, S.H. Optimization of distribution planning for agricultural products in logistics based on degree of maturity. Comput. Electron. Agric. 2019, 160, 1-7. [CrossRef]

24. Cao, Q.; Shao, J.P.; Sun, Y.A. Multi-objective fresh agricultural product distribution path optimization based on improved genetic algorithm. Ind. Eng. J. 2015, 15, 71-76.

25. Choi, K.; Lee, G.; Han, Y.J.; Bunn, J.M. Tomato maturity evaluation using color image analysis. Trans. ASAE 1995, 38, 171-176. [CrossRef]

26. Shiue, Y.C. An inventory model for perishable items in a lot-size system with quantity discounts. Eur. J. Oper. Res. 1990, 45, 260-264. [CrossRef]

27. Savelsbergh, M.W.P. Local search for routing problem with time windows. Ann. Oper. Res. 1985, 35, $254-265$. [CrossRef]

28. Zheng, J. Research on food vehicle routing problem based on improved genetic algorithm. J. Food. Sci. Technol. 2015, 8, 219-222. [CrossRef]

29. Wang, K.; Lan, S.; Zhao, Y. A genetic-algorithm-based approach to the two-echelon capacitated vehicle routing problem with stochastic demands in logistics service. J. Oper. Res. Soc. 2017, 68, 1409-1421. [CrossRef]

30. Yang, B.; Hu, Z.H.; Wei, C.; Li, S.Q.; Zhao, L.; Jia, S. Routing with time-windows for multiple environmental vehicle types. Comput. Ind. Eng. 2015, 89, 150-161. [CrossRef]

31. Pierre, D.M.; Zakaria, N. Stochastic partially optimized cyclic shift crossover for multi-objective genetic algorithms for the vehicle routing problem with time-windows. Soft Comput. 2016, 52, 863-876. [CrossRef]

32. Holland, J.H. Adaptation in Natural and Artificial Systems: An Introductory Analysis with Applications to Biology, Control, and Artificial Intelligence; MIT Press: Boston, MA, USA, 1992. 